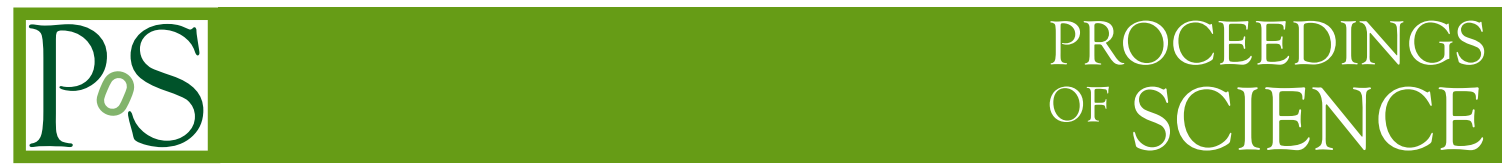

\title{
Gauge field mass problem in new aspect
}

\section{Nelly Konopleva* $\dagger$}

"VNIIEM" Corporation" JSC, Moscow, Russia

E-mail: nellyetheor.jinr.ru

Different interpretations of Higgs Lagrangian are under discussion. It is shown that Higgs boson can be not only elementary particle, but complex system or topological characteristic also. It is pointed out necessity of the new experiment schemes corresponding with each of hypotheses about Higgs boson mass nature which is being propound now.

XXI International Baldin Seminar on High Energy Physics Problems

September 10-15, 2012

JINR, Dubna, Russia

\footnotetext{
${ }^{*}$ Speaker.

${ }^{\dagger}$ It was supported by Organizers of ISHEPP XXI
} 
It is well known that gauge field mass can be produced by Higgs mechanism and spontaneous breaking of the gauge field theory symmetry. But physical sense of Higgs objects is undeterminate. So, the question appears: are Higgs bosons real particles or not? In supersymmetrical models it is so, but other interpretations of the Higgs mechanism are possible.

For example, in the pure Higgs model, which contains only Higgs bosons, the solutions of the classical field equations exist, which describe magnetic monopoles of Dirac type. It is possible to find as singular as regular solutions. It would be very interesting to detect at LHC Dirac's monopole instead of additional elementary particle of usual type.

Other variant of nonstandard interpretation of Higgs mechanism is connected with spontaneous breaking of the local gauge symmetry. As a result of this breaking the structure of vacuum changes. The singularities and multi-vacuum structure can arise. Plurality of vacuums prevent exact interpretation of the results of elementary particle interactions. One of the variants leads to the field algebra and vector dominance. In this case Higgs bosons are not real elementary particles. They are connected with topological properties of the space-time and group parameters space.

Very interesting situation arises when Higgs Lagrangian is regarded as analog of GinzburgLandau Lagrangian in superconductivity. Then Higgs mechanism becomes the analog of mechanism generating Cooper's pairs. In this case the Higgs bosons are the analog of Cooper's pairs, i.e. complex system. Which experiments can the Higgs boson mass be measured in? As Cooper's pairs exist only inside superconductor, such experiments must be done inside superconductor also, i.e. in some media. But measurements of elementary particle masses are usually fulfiled on a long distance from the vacuum domain, where these particles exist and interact. Are these experiments adequate to physical sense of the problem? Can Higgs boson mass be determined in the experiments of such type, if Higgs boson is complex system? Cooper's pairs do not exist outside superconductor.

So, must Higgs bosons be real elementary particles or they are complex systems or auxiliary mathematical objects - these are the main questions of modern theoretical and experimental elementary particle physics. It is necessary to propose new schemes of the experiments to choose between the above different hypotheses about Higgs boson mass nature. 